\title{
Great balls of fire and the vicious cycle: A study of the effects of cycling on male fertility
}

\author{
Tom Southorn, BSc, Fourth Year Medical Student, St George's Hospital Medical School, University of London, Cranmer Terrace, \\ London SW17 ORE, UK. E-mail: 97ms144@sghms.ac.uk
}

Correspondence: Tom Southorn, 87 Kenlor Road, Tooting, London SW17 0DG, UK. E-mail: tsouthorn@hotmail.com

The Journal of Family Planning and Reproductive Health Care 2002: 28(4): 211-213

\begin{abstract}
Over the past few years we have been bombarded with publicity telling us to do more exercise in order to reduce our risk of developing heart disease. Also, as commuter traffic increases and petrol prices rise, workers are constantly looking for quicker, cheaper and greener ways of travelling short distances. As a result of this, bicycle sales have risen exponentially. However, as the popularity of cycling increases, so do the fears that spending hours in the saddle every day may not be as beneficial as first thought. For many years now reports in the literature have suggested that exercise in general, and cycling specifically, may actually increase an individual's risk of developing problems in the male reproductive system. In this report I will review the evidence available in the literature, paying special attention to cycling and the risks of developing testicular cancer, secondary impotence and, most importantly, the effects on male fertility.
\end{abstract}

\section{The effects of exercise on potency and fertility \\ Testicular cancer}

There has been a lot of work done recently on the aetiology of testicular cancer and any predisposing or protecting factors. In England and Wales testicular cancer is now the most common form of cancer in men aged 15-44 years, ${ }^{1}$ and over the past few decades there has been a continuous rise in the incidence. The identification and modification of risk factors could stem this rise and even cause a decrease in the number of new cases. At present the only risk factors that have been identified with certainty are a history of undescended testis and infantile inguinal hernia.

Forman et al. ${ }^{1}$ undertook a study to determine the risk of testicular cancer associated with undescended testis, inguinal hernia, age at puberty, marital status, infertility, vasectomy, and amount of exercise. The key findings associated with exercise were that the risk of testicular cancer decreased with increased amount of exercise (over 15 hours a week) and increased with increased sedentary time. Subfertile men are known to have an increased risk of developing testicular carcinoma in situ, ${ }^{1}$ and it has been suggested that cancer and subfertility are both associated with an underlying defect in spermatogenesis. The common finding of a reduced sperm count in men with testicular cancer ${ }^{1}$ strengthens this hypothesis. It is thought that large amounts of exercise might alter hormonal profiles and that these hormones might exert some effect on the rate of mitosis of germ cells, leading to a decreased risk of developing testicular cancer. ${ }^{1}$

In an earlier study, Coldman et al. ${ }^{2}$ looked at the relationship of testicular seminoma and particular activities in addition to cryptorchidism and infantile inguinal hernia.
In particular they looked at the possible role that trauma might play in the aetiology of testicular cancer. Trauma may well act as a trigger for diagnosis, but can it also predispose an individual to develop testicular cancer? The authors found a relationship between testicular cancer and cycling and horse riding. Both of these activities may expose the scrotum to direct and repeated trauma, which is unlikely to be encountered in any other situations. Both activities also involve close contact with leather or similar products, which may have been treated with a large variety of chemical dyes and waterproofing agents.

\section{Nerve entrapment, impotence and subfertility}

Damage or entrapment of the pudendal nerves and/or arteries is a well-documented complication of cycling, although mainly from case reports. ${ }^{3-5}$ In the majority of these case reports, the cases presented with penile insensitivity following lengthy bike rides. Symptoms also included bladder outflow obstruction ${ }^{4}$ and secondary impotence, which may persist for weeks, even months. ${ }^{5}$ It is thought that the pudendal nerves are trapped between the nose of a narrow bicycle saddle and either the pubic bone ${ }^{5}$ the pubic symphysis ${ }^{3}$ or the ischial bone. ${ }^{6}$ The symptoms of bladder outflow obstruction are thought to be as a result of compression of the proximal urethra, external urinary sphincter and prostate, also caused by the nose of the bicycle saddle. ${ }^{4}$ In almost all cases, reduction or cessation of cycling activities resulted in full recovery from symptoms. For those continuing to cycle long distances, accurate set-up of the bicycle with regard to saddle height in relation to handlebar height, and the use of a wider, more padded seat would seem to alleviate the symptoms in most cases.

Aboseif et al. ${ }^{7}$ investigated the effects of occlusion of the internal pudendal and penile arteries on the erectile response of dogs. They found that acute occlusion of one artery did not affect the response, as the contralateral artery compensated with an increase in flow. However, bilateral occlusion significantly reduced the response. Erection was not abolished completely due to the presence of small collateral arteries, which were not blocked. Chronic bilateral occlusion does not significantly affect erection, as these collaterals develop to take the place of the occluded arteries. The acute occlusion effects on dogs possibly mimic the effects that cycling may have on human erectile function.

The term 'Alcock syndrome' was first established in 1987 by Amarenco (reviewed by Oberpenning ${ }^{6)}$ to describe this type of cyclist paralysis. The pudendal nerve originates from the sacral plexus and circulates around the sacrospinal ligament and subsequently enters into the fatty tissues of the 
ischiorectal fossa. Close to the insertion of the ischiotuberal ligaments into the ischial bone the pudendal nerve enters the Alcock canal, accompanied by the pudendal artery and vein. After passage through this canal, the pudendal nerve continues its course, emerging from below the symphysis to provide innervation of the perineum and genitals. Sitting on a hard, narrow bicycle saddle, together with the mechanical stress of rotating the pedals, places a great deal of stress on the perineum and the Alcock canal and, as a result, nerve and arterial compression against the bones of the pelvis occurs. Downward pointing, or wider bicycle saddles, may alleviate this problem.

Andersen et al. ${ }^{8}$ examined the association between cycling and symptoms of nerve entrapments. Their study looked at participants of an annual cycling race in Norway. The Great Trial of Strength is a non-stop 540-km race from Trondheim to Oslo. The authors distributed questionnaires addressing several factors as detailed below to those competing:

1. Training frequency before the tour

2. Average number of weekly tours of at least 1 hour during the past 3 months

3. Number of previous long rides of more than $100 \mathrm{~km}$

4. Number of previously completed tours of the Great Trial of Strength.

Symptoms were assessed under four headings as follows:

1. Hand weakness

2. Numbness in the hands

3. Numbness in the genital region

4. Impotence.

Libido and ejaculation were not assessed. Cyclists complaining of symptoms were followed up for further details. (The results for nerve entrapment in the hands are not relevant to this discussion and so are not mentioned further here.)

It is thought that the incidence of impotence in cyclists is very low, although still higher than in the healthy population. ${ }^{5}$ Obtaining reliable data on this subject is very difficult so the values quoted by Andersen et al. ${ }^{8}$ (impotence 13\%) must be regarded as minimal values. From this study it is suggested that long-lasting pressure of 1-2 days is needed to induce symptoms of impotence. It is thought that the pathophysiological process is a combination of ischaemic neuropathy, arterial insufficiency and nerve compression.

This idea of a connection between long-standing pressure from the saddle and impotence is not a new one. Hippocrates first documented evidence of this phenomenon, although not in cyclists! Writing about the Scythians, a caravan people living north of the Black Sea and renowned for their equestrian skill, he mentioned that 'The constant jolting on their horses unfits them for intercourse'. He also states that 'These complaints come upon the Scythians and they are the most impotent of men'. Hippocrates states that this affliction is also due to the fact that 'They always wear trousers and spend most of their time on their horses, so that they do not handle the parts, but owing to cold and fatigue forget about sexual passion'. 8

In general, Andersen et al. ${ }^{8}$ state that an ischaemic cause of nerve block is expected to be of shorter duration than a demyelinating cause due to pressure. In long cycling tours, an uninterrupted period of 10-15 hours on the bike is not uncommon, and duration of symptoms seems to be related to the number of hours spent continuously in the saddle. If demyelination does occur, symptoms may persist for up to 6 months. $^{8}$
Torsion

Jackson and $\mathrm{Craft}^{9}$ state, in a case study, that cycling may be a significant cause of torsion of the testis. They describe five cases of teenage boys presenting with torsion, all of whom described the onset of pain as starting during or shortly after completion of a bike ride. It would appear that the testis could become twisted between the thigh and the saddle as the legs go up and down. If the torsion is not corrected quickly, orchidectomy is indicated to remove the dead testis. This may lead to a significant effect on fertility in later life.

\section{Scrotal temperature}

It is a commonly accepted belief that tight-fitting underwear could be responsible for the recent decline in male fertility. The reason for this being that tight-fitting underwear is believed to lead to a rise in scrotal temperature. Davidson first recognised the potential for underwear type to influence scrotal temperature and fertility in $1954 .{ }^{10}$ In a recent study by Munkelwitz and Gilbert ${ }^{10}$ they found no significant difference in scrotal temperatures between men wearing brief-style underwear and men wearing boxer shorts. Could this belief be a myth? It is certainly based on very little actual evidence. Strong evidence remains, however, that raising scrotal temperature results in atrophy of the testicular germinal epithelium and arrest of spermatogenesis. ${ }^{10}$

Arce et al. ${ }^{11}$ suggested that the effects of exercise on spermatogenesis might not be as a result of hormonal changes but might, in fact, be as a result of increased scrotal temperature. This increase may be as a result of wearing tight, supportive clothing during periods of strenuous exercise. It has been shown in other studies that elevations in scrotal temperature beyond 30 minutes may result in disturbing effects on spermatogenesis. Lucía et al. ${ }^{12}$ suggested that this might be of special relevance to the issue of subfertility and cycling, given that professional cyclists exercise for several hours a day wearing close-fitting Lycra outfits, which may lead to a marked and sustained rise in scrotal temperature.

\section{Discussion}

Is exercise really good for you?

Besides considerable health benefits from exercise, the increased pursuit of fitness also results in some health hazards with which the physician must deal. Cycling is certainly no exception, and may actually be worse than many other forms of exercise when performed at a high level. The studies reviewed here show a few different mechanisms for how exercise, in particular cycling, might affect male health and fertility.

\section{Testicular cancer}

These studies suggest that exercise at a reasonably high level has a protective effect against testicular cancer. However, cycling, as well as horse riding, seem to predispose an individual to testicular cancer. It is impossible to say whether these two facts might cancel each other out, however it is certainly a fact that could be explored further. More work would need to be done looking at cyclists and horse riders in particular. It is worth noting that Lance Armstrong, who has won the Tour De France cycle race, reputedly the toughest race in the world, for the last 2 years, was diagnosed as having testicular cancer with metastases. Fortunately, he made a full recovery before astounding everyone by winning the race for the first time against all odds. Armstrong was advised to freeze a sperm sample 
before commencing his chemotherapy and has since fathered a child. This would suggest that even cycling at a professional level does not reduce your fertility enough to make you unable to fertilise an egg. However, the question still remains as to whether Armstrong developed the disease as a result of the sheer amount of gruelling training needed to be a professional cyclist?

\section{Nerve entrapment and impotence}

It would seem from these studies that cycling does indeed cause neuropathy, however, it still seems unlikely that this has any long-term effect on male fertility. The most severe effect would seem to be loss of penile sensation for a period of time, related to the extent of injury. Researchers seem to be divided as to whether cycling can cause impotence. Andersen et al. ${ }^{8}$ provide good evidence to say that it can, in some individuals. Due to the personal nature of this problem, it is worth remembering that many sufferers may not wish to disclose the information to a researcher or clinician and so the problem is almost certainly underreported. Similar studies performed at other endurance cycling events may provide further good evidence on this problem. An interesting point would be to study the differences in development of symptoms between those cyclists who partake in road races, where the majority of the ride is spent firmly in the saddle, and those who ride in offroad mountain bike races. Due to the more technical nature of mountain bike racing, the rider will tend to shift his body weight on and off the saddle at regular intervals.

\section{Trauma}

It is accepted that long-distance cycling results in testicular trauma that would not be experienced in many other pursuits. It remains to be tested how this trauma might affect male fertility. This is a hard variable to study as trauma is impossible to measure accurately in a retrospective study, and a prospective study is hampered by the very real problem that volunteers would be very unwilling to subject themselves knowingly to testicular trauma, no matter how small.

\section{Increased temperature}

The long periods of activity associated with high-level exercise, especially cycling, will undoubtedly cause an increase in core body temperature. It would also seem to cause an increase in scrotal temperature, especially if tight, supportive clothing is worn at the same time. This increase, if prolonged, is likely to cause deleterious effects on spermatogenesis. How severe these effects are is a subject that the many hundreds of young professional cyclists would like to be explored before too much longer. Until then, their profession dictates that they don their brightly coloured, sponsor-covered, Lycra race suits for every race.

\section{Conclusions}

There is a vast amount of literature available on the subject of exercise and male fertility. What is reviewed here is only a tiny fraction. However, even from these studies it is clear that there is potential for much more work to be done on this subject. The question ' $C$ an exercise result in a reduction in male fertility?' has not yet been fully answered. Even in the light of all this research, cycling is enjoying its most popular period ever, and the public is still being told that the more exercise you do, the healthier you will be and the longer you will live. This project suggests that in some cases this may not be true. The association between cycling and urogenital pathology has not gone unnoticed by the bicycle industry.
At present there are a vast number and range of saddles on the market, utilising novel designs and materials in an attempt to relieve pressure on the more sensitive parts of the perineum and instead take advantage of the bony skeleton. Whether any of these new designs proves to be successful remains to be seen. In the meantime it is encouraging to see the cycling industry keeping up to date with current research.

References

1 Forman D, Pike MC, Davey G, et al. Aetiology of testicular cancer: association with congenital abnormalities, age at puberty, infertility and exercise. BMJ 1994; 308: 1393-1399.

2 Coldman AJ, Elwood JM, Gallagher RP. Sports activities and risk of testicular cancer. Br J Cancer 1982; 46: 749-755.

3 Goodson JD. Pudendal neuritis from biking. N Engl J Med 1981; 304: 645

4 O'Brien KP. Sports urology: the vicious cycle. N Engl J Med 1981; 304: $1367-1368$.

5 Desai KM, Gingell JC. Hazards of long distance cycling. BMJ 1989; 298: $1072-1073$.

6 Oberpenning F, Roth S, Leusmann DB, et al. The Alcock syndrome: temporary penile insensitivity due to compression of the pudendal nerve within the Alcock canal. J Urol 1994; 151: 423-425.

7 Aboseif SR, Breza J, Orvis B, et al. Erectile response to acute and chronic occlusion of the internal pudendal and penile arteries. J Urol 1989; 141: 398-402.

8 Andersen KV, Bovim G. Impotence and nerve entrapment in long distance amateur cyclists. Acta Neurol Scand 1997; 95: 223-240.

9 Jackson RH, Craft AW. Bicycle saddles and torsion of the testis. Lancet 1978; 1: 983-984.

10 Munkelwitz R, Gilbert B. Are boxer shorts really better? A critical analysis of the role of underwear type in male subfertility. $J$ Urol 1998; 160: 1329-1333.

11 Arce JC, De Souza MJ, Pescatello LS, et al. Subclinical alterations in hormone and semen profile in athletes. Fertil Steril 1993; 59: 398-404.

12 Lucía A, Chicarro J, Pérez M, et al. Reproductive function in male endurance athletes: sperm analysis and hormonal profile. J Appl Physiol 1996; 81: 2627-2636.

\section{herpes simplex can be more than a physical problem ...}

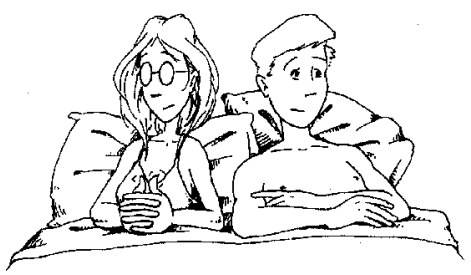

The Herpes Viruses Association helpline supplies information to patients and medical practitioners.

Helpline volunteers who have the virus, including 'pregnancy helpliners' who have given birth, are trained to counsel and advise.

* "Patients with genital herpes suffer considerable psychological morbidity and

the fact that they are able to receive very sophisticated and experienced

counselling ... has been extremely useful in helping them deal with thei condition. The HVA has fulfilled this role admirably over the years Prof. M W Adler MD FRCP FFPHM, UCL Medical School, London

* "Nobody does what they do, filling an important niche." Dr Paul Simmons MB FRCP, St Bartholomew's Hospital, London

\section{helpline 02076099061}

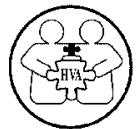

For leaflets, patient information cards or posters, write or send e-mail to:

HVA, 41 North Road, London N7 9DP marian@herpes.org.uk

We often hear this comment

"I wish l'd been told about you years ago" 